\title{
Eacilitatory Effects of Spectral Lights upon Unit Discharge Caused by Electrical Stimulation of the Cat's Retina
}

\author{
Yoshiyuki Imazawa \\ Department of Physiology (Prof. K. Motokawa)
and \\ Department of Orthopedics (Prof. S. Iino), \\ Tohoku University School of Medicine, Sendai
}

\begin{abstract}
Electrical stimulation of the retina causes discharges of ganglion cells. The responses may be divided into two classes on the basis of their latencies, that is, a short-latency response and a long-latency one. With an on-unit the short-latency response was evoked by a corneopositive current, but the longlatency one by a corneonegative current. With an off-unit a reversed relation was found between the latency and the direction of the current. All four kinds of responses were remarkably facilitated by steady focal illumination and their latencies shortened according to the increase of light strength except for a short latency of an off-unit which was prolonged with increasing intensity of light. In the present experiment the record was obtained from the optic tract of the cat. The dependence of electrical threshold and latency upon the wavelengths of monochromatic lights was determined, using steady focal illumination. The facilitatory effect of light upon the electrical threshold and the latency was found to be maximal in the neighborhood of $500 \mathrm{~m} \mu$, but some units showed exceptional behavior.
\end{abstract}

It has been generally accepted that the retina of the vertebrate is sensitive to electric current passing through it. On the basis of experimental results, structures stimulated by the transretinal current have been proposed to be located somewhere between the outer plexiform and inner nuclear layers. Such a proposal claims that electric and light stimulation would interact with each other.

Granit ${ }^{1,2}$ showed that the polarizing current passing through the cat's retina either depressed or enhanced light threshold of single retinal elements depending upon the character of response to light stimulation, although reversed current gave rise to opposite effects. Furthermore, Gernandt ${ }^{3}$ and Granit $^{4}$ reported that the sensitivity of elements to monochromatic light was also affected by the polarizing current, marked effects being found at blue, green and red regions in the spectrum.

In a series of experiments on the human eye, Motokawa and his co-workers ${ }^{5-7}$

Received for publication, May 31, 1966. 
showed that the electrical excitability of the eye, as determined by the reciprocal of threshold current required for just perceptible phosphene, was enhanced by pre-illumination with the time course characteristic of color used for the illumination. They also demonstrated similar effects in the retinas of the frog, ${ }^{8-10}$ the toad ${ }^{11,12}$ and the cat, ${ }^{13}$ using the discharge of ganglion cells as index.

Recently Taira, Imazawa and Motokawa, ${ }^{14}$ recording unit discharge from the cat's optic tract, showed that the occurrences of response of units to transretinal current pulses were markedly facilitated by steady focal illumination. In the present experiment facilitatory effects of monochromatic light upon electrically induced responses were measured and plotted against wavelengths. Most of the curves obtained were in quite good agreement with the human scotopic luminosity curve, except for humps or subpeaks at blue, green and red regions in the spectrum.

\section{MethoDs}

Experiments were performed on adult cats. Methods were basically similar to those used in the previous paper. ${ }^{14}$ All surgical procedures from the cannulation into the trachea to trepanation were carried out under ether anesthesia. The animal was fixed to a stereotaxic apparatus of Johnson type and a trephine hole of $13 \mathrm{~mm}$ in diameter was centered at $\mathrm{H} \mathrm{7.0,} \mathrm{L} 11.0$ on the left side of the skull. The hollow lucite peg filled with $6 \%$ agar gel was fitted into the trephine hole and cemented to the bone with dental wax after removing the dura. These procedures minimized the respiratory and pulsatile movements of the brain and allowed recordings from a single unit long enough. During the experiment no general anesthetics were used but pressure points and wound margins were infiltrated locally with $1 \%$ procaine solution. Throughout the experiment the animal was immobilized with gallamine triethiodide (Flaxedil) which was injected through a polyethylene cannula inserted into the saphenous vein and artificially ventilated. The rectal temperature was kept at $38^{\circ}-38.5^{\circ} \mathrm{C}$ by means of an electric heating pad. The pupils of both eyes were dilated with a few drops of $1 \%$ atropine solution and the corneae were protected from drying by contact lenses.

A ring-form silver electrode for stimulation of the retina was held in place in the conjunctivum of the eye and an indifferent silver electrode was placed at the frontal portion on the same side, so as to stimulate one eye only. Current strength was measured by the voltage drop generated through a resistance of 10 $\mathrm{k} \Omega$ inserted into the circuit of stimulation. A rectangular current pulse of $5 \mathrm{msec}$ in duration was used as stimulus in most cases. A glass capillary electrode filled with $3 \mathrm{M} \mathrm{KCl}$ was employed for recording. The microelectrode was inserted into the optic tract through a metal guide tube $2 \mathrm{~mm}$ in diameter and set stereotaxically. A reference electrode, a silver plate was held in place on the exposed skull. Most 
of units were recorded from the optic tract, but a few were taken from the lateral geniculate nucleus and the optic radiation fibers.

A screen of the frosted glass was placed at a distance of $60 \mathrm{~cm}$ from the eye of the animal and a stimulus light spot was projected on this screen. Light spots were circular in shape and below $0.8^{\circ}$ in visual angle. The maximum intensity of the light spot was 20 lux on the screen and the intensity was controlled by means of calibrated neutral density filters of which transmission factors were $0.7^{\circ}, 0.7^{1}$, $0.7^{2} \ldots$ The stimulus spot was focused anywhere on the screen by rotating plane mirror and projected on the center of the receptive field. The majority of recorded units were scattered within $10^{\circ}$ in visual angle from the area centralis. Colored lights of equal energy were obtained by the use of interference filters. The wavelength ranged from $400 \mathrm{~m} \mu$ to $680 \mathrm{~m} \mu$ in $20 \mathrm{~m} \mu$ steps.

Electrical stimulation was made every 2 seconds to avoid aftereffects produced by the preceding stimulation which usually lasted 1 second or so. All the experiments were performed in the condition of dark adaptation or under steady focal illumination, the area beside the portion of illumination being kept in the dark.

CCFs of several units were measured to evaluate the effectiveness of light in the retina, the CFF being defined as the minimum frequency of flickering light at which the strict relationship of flash and discharge ceased. ${ }^{15}$ They amounted about $49 \mathrm{cps}$ on an average at the maximum intensity. The flickering light was made by rotating a sectored disk placed in the light path.

\section{Results}

\section{1) Response patterns of the retina to photic and electrical stimulation}

Most fibers picked up from the optic tract responded with pure on- or offdischarges to photic stimulation as shown in Fig. $1, A$ and $A$ ' respectively. In the present paper, the term 'on' and 'off' will be used only for the responses to photic stimulation but not for those to electrical stimulation.

As shown in our previous paper, ${ }^{14}$ on- and off-units responded to electrical stimulation in different manners. The on-unit showed a short-latency response of burst discharge to an ingoing or corneopositive current (see Fig. 1, B) and a long-latency response to an outgoing or corneonegative current (Fig. 1, D). The latency of the former response was about $10 \mathrm{msec}$ and that of the latter about $30 \mathrm{msec}$ in the dark-adapted state. This relation is reversed with the off-unit as can be seen in Fig. 1, $B^{\prime}$ and $D^{\prime}$. It responded with a short latency of about $10 \mathrm{msec}$ to the corneonegative current and with a long latency of about 30 msec to the corneopositive current. The latency varied little with the change of current intensity, while it showed remarkable shortening when the brightness of the focal illumination centered on the receptive field was increased. The short-latency response of the off-unit was an exception to this rule. The latency 


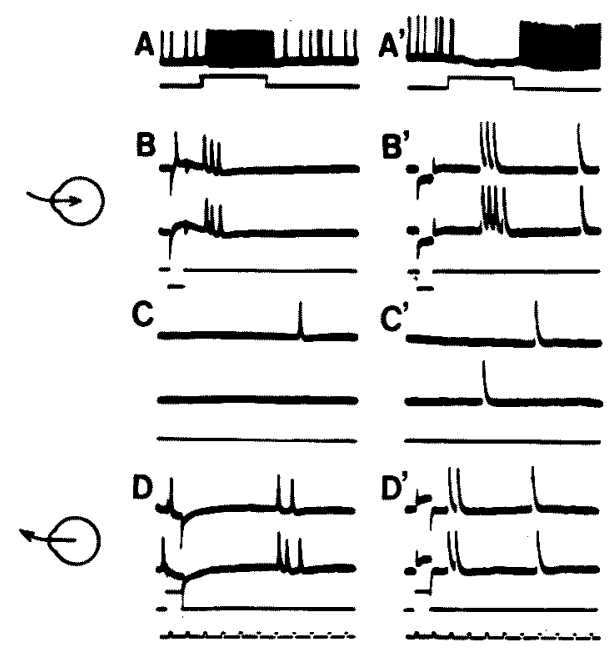

Fig. 1. Response patterns of on- and off-units to photic and electric stimuli. Traces in the left column are for on-unit and those in right for off-unit. $A$ and $A^{\prime}$ : responses to photic stimulation. Period of stimulation is shown by upward deflection in the lower trace. $B$ and $B^{\prime}$ : responses to comeopositive current pulse $(5 \mathrm{msec}$ in duration, $800 \mu \mathrm{A})$. The lowest trace shows the time of stimulation. $C$ and $C^{\prime}$ : unitary discharges when no electrical stimulation was given. $D$ and $D^{\prime}$ : responses to corneonegative current pulse. Time scale: $5 \mathrm{msec}$. The experiment was performed in the dark. To the left the direction of current is schematically shown.

was prolonged with an increase in the brightness of the focal illumination. In addition to such changes in latency, the threshold intensity of electric current for the responses was also affected by the focal illumination of the receptive field.

In passing, it will be convenient to mention the way of determining the threshold intensity of the electric stimulus. The detection of response discharges among spontaneous ones was a tricky business. So all spikes included over the period of $10 \mathrm{msec}$ within which the most response spikes seemed to appear were regarded as reponse. This naturally implies that the presence of vigorous spontaneous discharge makes the detection of the response difficult. The stimulating pulse was varied in intensity step by step and at each intensity three trials were made. The least strength of current which produced two positive responses in three trials were taken as the threshold. Such procedures were justified in view that $60 \%$ level is the steepest portion in the occurrence probability versus current strength curve as shown previously. ${ }^{14}$

2) Changes of electrical excitability under steady illumination of monochromatic light

As reported previously, ${ }^{14}$ the electrical threshold of both on- and off-units took a characteristic time course on a focal illumination of the receptive field 
until it settled at constant level. In view of this fact the electrical threshold was measured several seconds after each monochromatic light was turned on in this series of experiments.

As can be seen in Fig. 2, $A$, electrical thresholds were markedly lowered by illumination of monochromatic lights in comparison with that in the dark. The majority of recorded units had a minimum threshold at about $500 \mathrm{~m} \mu$. Some of them had an additional depression at $620 \mathrm{~m} \mu$. These results are comparable with the work of Okuda, Taira and Motokawa ${ }^{\mathbf{1 6}}$ in which the spike numbers of response were plotted against wavelengths. In one of the remaining units there was no such a minimum, but uniformly lowered threshold distributed over a wide range of wavelengths (Fig. 2, $B$ ). Some of other units had two well-marked minima as shown in Fig. $3, A$ and $B$. The minima were at 480 and $520 \mathrm{~m} \mu$ for the unit shown in Fig. 3, $A$, obtained from the optic tract and 460 and $520 \mathrm{~m} \mu$ for the unit shown in Fig. 3, $B$, obtained from a postsynaptic neuron in the LGN. These portions were repeatedly measured to avoid haphazard experimental errors. In the present experiment no unit was found which underwent a dominant facilitatory effect at yellow or red portions in the spectrum.

Fig. 4 presents an interesting finding in which considerably weak steady illumination exerted a suppressing effect contrary to a facilitatory effect of strong illumination. The electrical threshold of this unit in the dark was $1,120 \mu \mathrm{A}$. With a maximal intensity of the focal illumination it was greatly lowered, having a minimum at about $480 \mathrm{~m} \mu$ (Fig. $4, A$ ). When the intensity was reduced to $24 \%$ by means of a neutral density filter, the threshold was markedly enhanced, still having a minimum at $500 \mathrm{~m} \mu$ (Fig. $4, B$ ).

A

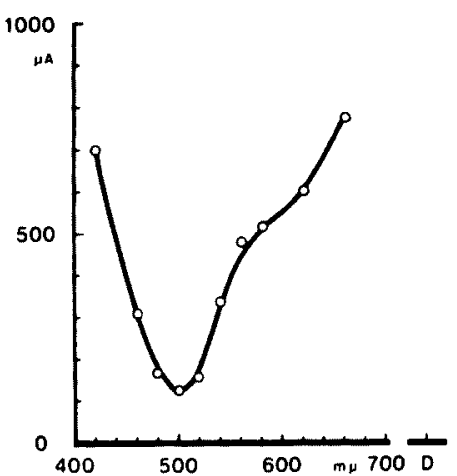

B

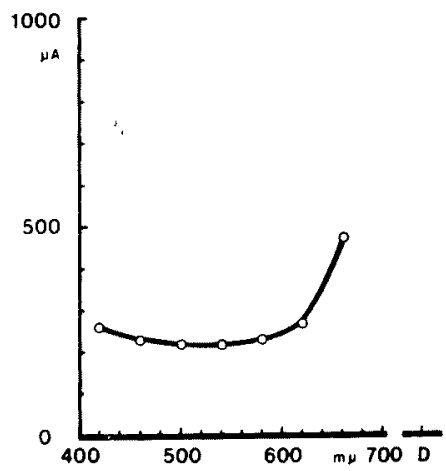

Fig. 2. Threshold strengths of electric current are plotted as ordinates against wavelengths of monochromatic light as abscissae. A was obtained for the short-latency response of off-unit to corneonegative current pulse, and $B$ for the short-latency response of on-unit to corneopositive current pulse. Letter ' $D$ ' stands for 'in the dark'. 
A

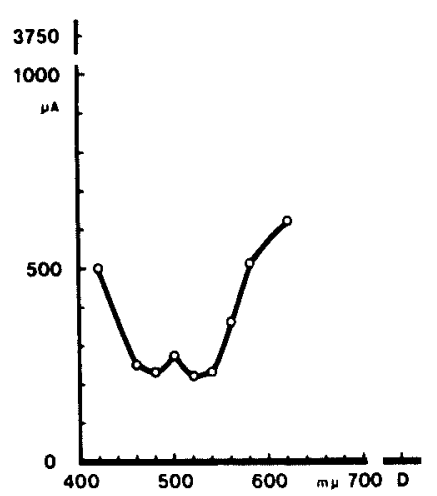

B

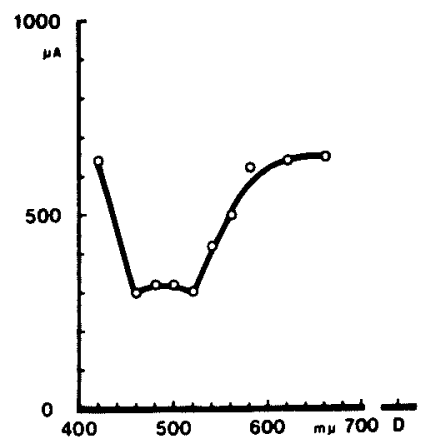

Fig. 3 Electrical thresholds vs. wavelengths. $A$ was obtained from off-unit in optic tract and $B$ from soma off-unit of lateral geniculate nucleus. Stimulus current was corneonegative for both $A$ and $B$. Further details are the same as in Fig. 2.

A

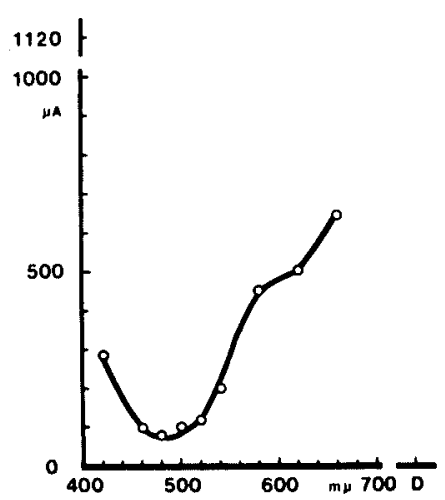

B

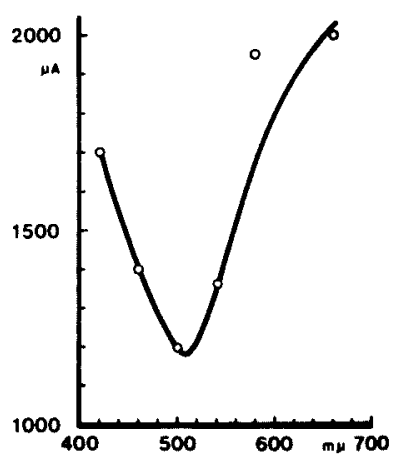

Fig. 4. Electrical thresholds at two different levels of light adaptation. Ordinates and abscissae are the same as in Fig. 2. Records were obtained from off-unit of optic radiation fiber. $A$ : electrical threshold vs. wavelength curve under steady focal illumination of 20 lux. $B$ : intensity of illumination was reduced to $24 \%$ of that of $A$. Stimulus current was corneonegative.

To compare all the curves mentioned above with one other on the same scale the following value was calculated for normalization of the curves. The relative enhancement of electrical excitability, $\zeta$ is defined by the formula

$$
\zeta=\frac{E-E_{0}}{E_{0}}
$$

where $E$ stands for the excitability (reciprocal of threshold current) during 
A

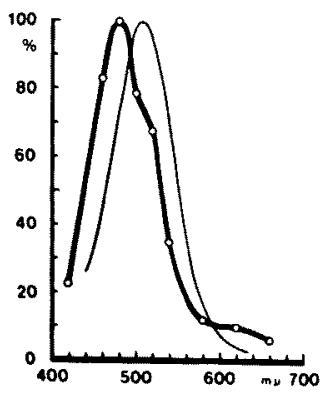

B

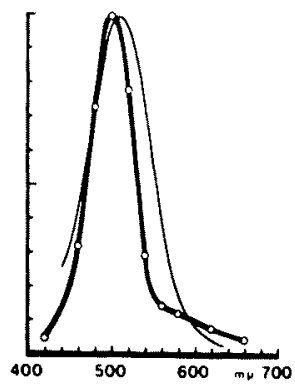

C

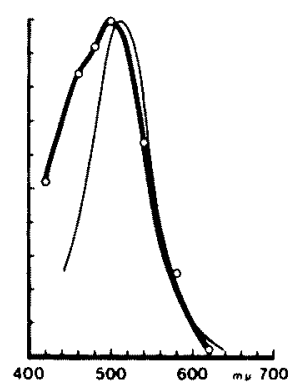

Fig. 5. Relative electrical excitabilities vs. wavelengths. Open circles indicate results obtained in this experiment and thin line stands for the human scotopic luminosity curve. A: on-unit. Current direction comeopositive. $B$ and $C$ : off-units. Current direction corneonegative. Ordinates: relative electrical excitability. Abscissae: wavelength. For further details see text.

illumination and $E_{0}$ for the excitability without illumination. The values of $\zeta$ were plotted as ordinates in per cent of the maximum against wavelengths. Fig. 5 illustrates these curves. Thick lines indicate the relative enhancement of electrical excitability obtained in the present experiments and thin lines the human scotopic luminosity curve ${ }^{17}$ drawn on the same scale. As can be seen in Fig. 5, $A$, the electrical excitability has a peak at $480 \mathrm{~m} \mu$ and a hump at $520 \mathrm{~m} \mu$, and in Fig. 5, $C$ a peak is at $500 \mathrm{~m} \mu$ and a hump at $460 \mathrm{~m} \mu$. In Fig. $5, B$ the curve has a peak at $500 \mathrm{~m} \mu$ but no hump. In the present experiment the curves of types $C$ and $B$ were most common and as to the peak-wavelength they are in quite good coincidence with the human scotopic luminosity curve. The curve type $A$ was rather rare, and it shows a significant difference from the human scotopic luminosity curve. It might reflect 'modulators' which Granit proposed in his extensive work on the dominator-modulator hypothesis. ${ }^{18,19}$

3) The effects of steady focal illumination of monochromatic light upon the latency As described previously, ${ }^{14}$ in a fixed condition of the electrical stimulation, the fluctuation of latency of both the short-latency and the long-latency responses was considerable in the dark, while it was stabilized on illumination.

An off-unit, as illustrated in Fig. 6, had an active spontaneous discharge in the dark $(B)$ and gave a short-latency response to a corneonegative current pulse $(A)$ and a long-latency response to corneopositive current $(C)$. These responses were hardly detectable against the ongoing background discharge. When the focal steady light centered on the receptive field was turned on, the ongoing discharge was so completely suppressed $\left(B^{\prime}\right)$ that the electrically induced response became distinct. The fluctuation of latency of the responses was now negligible and also the long latency was shortened by as much as $10 \mathrm{msec}$ 
A

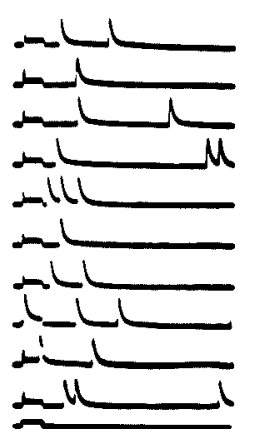

A'

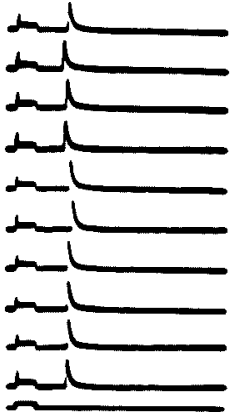

B

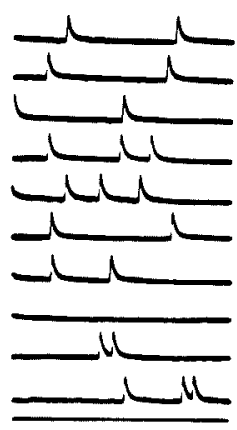

B'

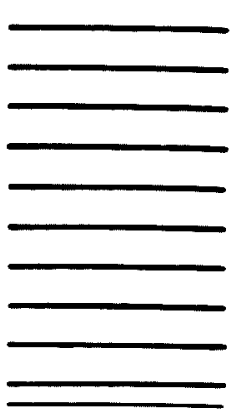

C

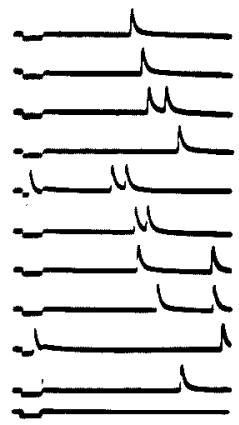

C'

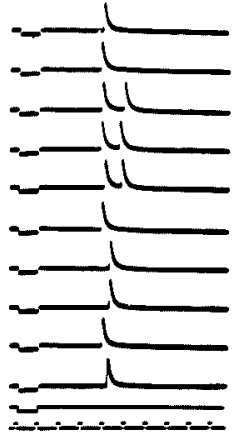

Fig. 6. Short-latency and long-latency responses of off-unit in the dark and during illumination. $A$ : short-latency responses. $B$ : control without electrical stimulation. $C$ : long-latency responses. All photographs were obtained in the dark. A', B' and $C^{\prime}$ : as in $A, B$ and $C$ but during illumination. Stimulus current was $800 \mu \mathrm{A}$ in intensity and $5 \mathrm{msec}$ in duration. Note the decrease of fluctuation of the latencies during illumination.

compared with that in the dark. In general the long latency underwent more striking reduction on the focal illumination. Hence, to study the latencyshortening effects of monochromatic light of different wavelength, the longlatency was mainly measured for a quantitative estimation of the effects. With fixed condition of electrical stimulation latencies were measured and averaged over twenty responses for each wavelength of monochromatic light.

Fig. 7 shows two examples of a plot of latency against wavelength in which a standard deviation (S.D.) is also indicated by a short vertical line to represent fluctuation of latency. An off-unit illustrated in Fig. 7, $A$ had the shortest latency of about $17.7 \mathrm{msec}$ at $500 \mathrm{~m} \mu$ (cf. $26.7 \mathrm{msec}$ control in the dark). Another off-unit shown in Fig. $7 B$ had the shortest latency of about $18.7 \mathrm{msec}$ at 580 $\mathrm{m} \mu$ (cf. $27.7 \mathrm{msec}$ in the dark). In these two and other units there was a general trend that the standard deviation was the smaller, the shorter the latency 

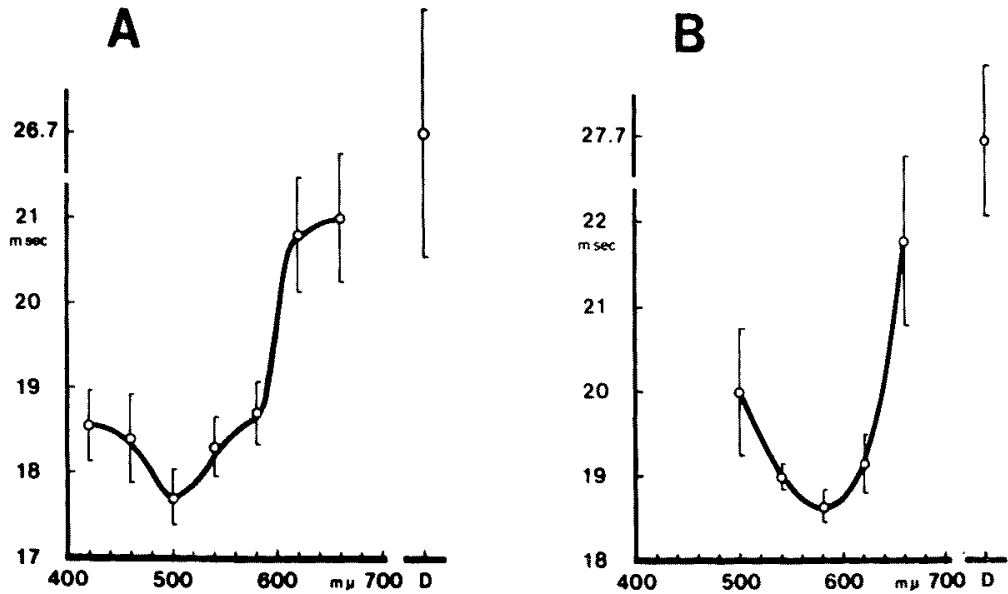

Fig. 7. Latencies vs. wavelengths. $A$ and $B$ were obtained from two different off-units. Ordinates: latencies for both $A$ and $B$. Abscissae: wavelengths. Letter ' $D$ ' indicates 'in the dark'. Stimulus current was $2 \mathrm{~mA}$ in intensity and 5 msec in duration, current direction being corneopositive for both $A$ and $B$. Vertical lines represent standard deviations.
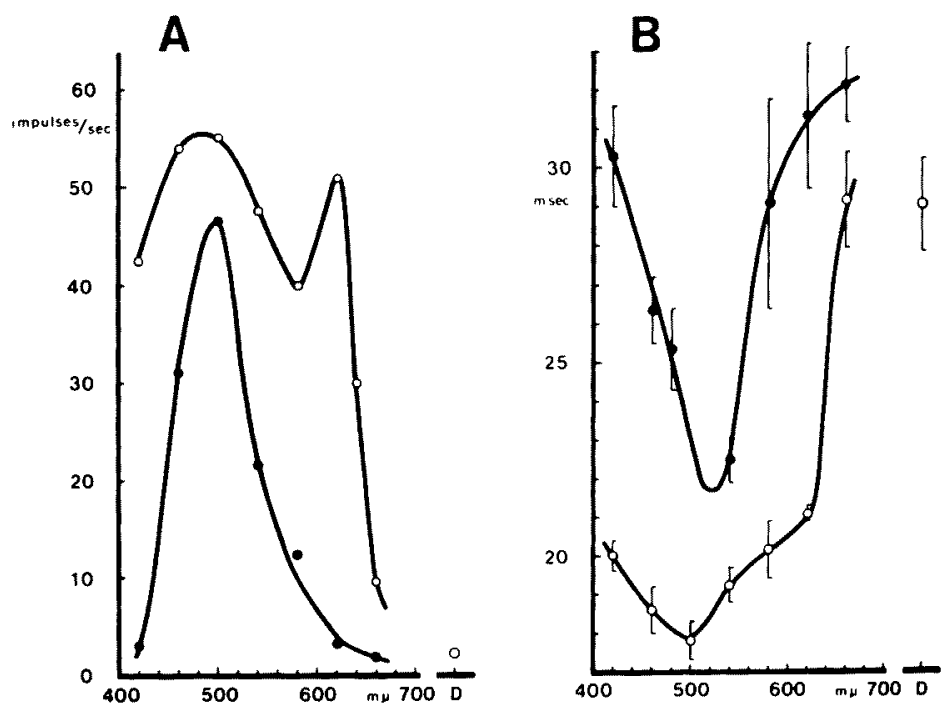

Fig. 8. Relation between discharge rate and latency of on-unit. $A$ : discharge rate vs. wavelengths. Ordinates: impulse numbers. Abscissae: wavelengths. $B$ : latencies of long-latency response. Ordinates: latencies. Abscissae: wavelengths. Stimulus current $(2 \mathrm{~mA}$ and $5 \mathrm{msec})$ was corneonegative. Open circles represent results obtained during illumination of 20 lux for both $A$ and $B$, and flled circles during illumination reduced to $24 \%$. Letter ' $D$ ' stands for 'in the dark.' 
became. Especially the decrease of S.D. was remarkable at wavelengths where the cone activity seemed to be enhanced.

Here a question will be raised whether thus obtained spectral latency curves have something to do with a spectral response curve in which spike number per sec is plotted against wavelength. Fig. $8, A$ shows spectral response curves obtained with an on-unit at two different intensities of monochromatic light. At lower intensity the spectral response curve (black dots) has only one prominent peak at $500 \mathrm{~m} \mu$. At higher intensity the discharge rate was accelerated over each wavelength and two distinct peaks (at 500 and $620 \mathrm{~m} \mu$ ) appeared in the spectral response curve (open circles). In this experiment the number of impulses was counted over a period of $5 \mathrm{sec}$ when the discharge rate had subsided to a steady level after a transient effect of changing light.

At two different levels of steady illumination the latencies of the electrically induced responses were also measured with one and the same unit and plotted against wavelengths. The lower curve thus obtained had only one peak at about $500 \mathrm{~m} \mu$ and a depression at $620 \mathrm{~m} \mu$ which corresponded to the subpeak seen in the impulse number vs. wavelength curve. It should be noted that the S.D. of latency was very small at $620 \mathrm{~m} \mu$.

\section{Discussion}

It has not yet been determined what structure is stimulated by the transretinal current. However, as suggested previously ${ }^{14}$ there have been some indications that the retinal network intervening between receptors and retinal ganglion cells is responsible for electrical excitation.

(1) The latency to the first spike of the electrically induced discharge of retinal ganglion cells was usually too long (about $10 \mathrm{msec}$ for short-latency response, about $30 \mathrm{msec}$ for long-latency one) to regard the discharge as resulting from direct stimulation of ganglion cells. Only with a very strong stimulus a single spike with short latency, about $2-4 \mathrm{msec}$, was evoked in addition. This single spike has been considered to result from direct stimulation of a ganglion cell, for the latency is comparable with the time required to cover the whole distance from the ganglion cell to the recording site with the mean conduction velocity of optic nerve fibers.

(2) Electrically induced response generally consisted of a succession of grouped discharges of several spikes, each group being separated by a silent period of about $25-50$ msec.

(3) Stimulating current of a given direction evoked two different types of discharge patterns depending upon response types of units concerned with photic stimulation. The corneopositive direction of stimulating current induced a short-latency $(10 \mathrm{msec})$ response in on-units and long-latency $(30 \mathrm{msec})$ one in off-units. The corneonegative current produced a long-latency response in on- 
units and short-latency one in off-units.

(4) Focal light-adaptation of the retina enhanced the electrical excitability of the retina or shortened the latency of the response.

(5) Periodical fluctuation of the electrical excitability was observed after subliminal stimulation with a 5 msec electric pulse.

The structures electrically stimulated have been discussed by Brindley ${ }^{20}$, Mita et al. ${ }^{21}$ in human experiments, Granit,, Motokawa et al. ${ }^{22}$ Crapper and Noell ${ }^{23}$ and Ogden and Brown ${ }^{24}$ in animal experiments.

From his experiments on the human eye, Brindley ${ }^{20}$ suggested that the structures stimulated may be bipolar cells or the parts of rod and cone cells lying inside the external limiting membrane. Mita et al. ${ }^{21}$ also in the human eye showed that receptor cells could be selectively stimulated by the $3 \mathrm{cps}$ alternating current and that with higher frequency of alternating current bipolar cells could be stimulated.

Motokawa et al. ${ }^{22}$ recorded a slow negative potential change in neighborhood of the external plexiform layer on direct stimulation of the isolated carp's retina. Ogden and Brown ${ }^{24}$ also recorded similar slow potential change by transretinal current stimulation. In all these discussions the structures intervening between retinal ganglion cells and the external limiting membrane are regarded as a possible site of electrical stimulation. Therefore, it will be safe to describe such structures with a noncommital term 'retinal network.'

In the present experiment the latency to the first spike of the electrically induced discharge was used as one of response measures in the retinal network. With on-units both short and long latencies were observed to decrease almost monotonically with an increase in intensity of the steady focal illumination within a range of intensity in this experiment. ${ }^{14}$ This would make us expect that if equally energized monochromatic light was used for the focal illumination instead of white light, the latency of the electrically induced response would alter depending upon physiological intensity of each monochromatic light.

In Fig. 8, spectral response curves and spectral latency curves obtained from the same unit have been compared with each other. Peaks in the spectral response curves correspond to either a minimum or a depression at the corresponding wavelengths in the spectral latency curve. It is to be noted that the doublepeak contour of the spectral response curve, obtained in stronger focal color adaptation clearly agreed with the contour consisting of a minimum and a depression at the same wavelengths in the spectral latency curve. The secondary peak appearing at $620 \mathrm{~m} \mu$ in the stronger illumination might represent the cone activity mediating the red process or red modulator in Granit's term.

Cat's retina is known to be predominated by rods and sparse in cones. Consequently most of retinal ganglion cells are expected to be largely controlled by an activity of the rod system. However, as suggested by Granit, ${ }^{19}$ few gan- 
glion cells which show modulator activity under light adaptation might be controlled by the activity of the cone system if favorable conditions were introduced.

To detect the cone activity in the cat's retina several procedures were used: (1) intermittent light stimulation at a high frequency, and (2) strong light adaptation, etc. Granit used strong light adaptation in attempts to isolate cone activity or modulators. Practically it was impossible for him to determine threshold intensities of colored light under stronger light-adaptations. Consequently he carried out measurements during initial phase of dark adaptation after extinction of adapting light. Measurements of latency of the electrically induced response have some advantages in carrying out experiments under steady illumination.

Measurements of threshold intensity of electrical stimulus for evoking a noticeable response allow us to estimate the excitability of the retinal network. The majority of curves representing the threshold intensities plotted against wavelengths used for steady focal illumination was in close agreement with the human scotopic luminosity curve having a minimum at $500 \mathrm{~m} \mu$. Under illumination of monochromatic light used in the present experiment, some units showed a secondary minimum or depression at longer wavelengths. Such a secondary minimum would be attributed to the activity of modulators which have been demonstrated in mammals by Granit.

The threshold method mentioned in the text has also advantages to enable measurements under steady illumination.

In the present report the number of units analyzed with these methods was not large enough, so that further experiments will be necessary.

\section{Acknowledgment}

The author wishes to thank Prof. K. Motokawa, Dr. N. Taira and Dr. T. Ogawa for their invaluable discussion and suggestion throughout the course of the experiment and the preparation of the manuscript.

\section{References}

1) Granit, R. The distribution of excitation and inhibition in single fibre responses from a polarized retina. J. Physiol. (Lond.), 1946, 105, 45-53.

2) Granit, R. Neural organization of the retinal elements, as revealed by polarization. J. Neurophysiol., 1948, 11, 239-252.

3) Gernandt, B. Color sensitivity, contrast and the polarity of the retinal elements. J. Neurophysiol., 1947, 10, 303-308.

4) Granit, R. The mammalian colour modulators. J. Neurophysiol., 1948, 11, 253-259.

5) Motokawa, K. Retinal processes and their role in color vision. J. Neurophysiol., $1949,12,291-301$.

6) Motokawa, K. Physiological induction in human retina as basis of color and brightness contrast. $J$. Neurophysiol., 1949, 12, 475-488. 
7) Gebhard, J.W. Motokawa's studies on electric excitation of the human eye. Psychol. Bull., 1953, 50, 73-111.

8) Motokawa, K. \& Iwama, K. Color receptors in frog eyes as revealed by electrical stimulation. Tohoku J. exp. Med., 1949, 50, 240.

9) Motok.wa, K. \& Iwama, K. The three color processes in the retina of frog. Tohoku $J$. exp. Med., 1949, 50, 258.

10) Motokawa, K. \& Iwama, K. Color processes and physiological induction in frog's retina. Tohoku J. exp. Med., 1951, 53, 341-349.

11) Motokawa, K., Iwama, K. \& Tukahara, S. Color processes in single retinal elements. Tohoku J. exp. Med., 1951, 53, 399-406.

12) Tukahara, S. Modulators and dominators of the toad's retina. Tohoku J. exp. Med., 1951, 54, 11-20.

13) Motokawa, K., Iwama, K. \& Ebe, M. Retinal color processes in cats. Jap. J. Physiol., 1952, 2, 198-207.

14) Taira, N., Imazawa, Y. \& Motokawa, K. Electrical stimulation of on- and offunits in cat's retina. Tohoku J. exp. Med., 1965, 85, 89-104.

15) Enroth, C. The mechanisms of flicker and fusion studied on single retinalelements in the dark adapted eye of the eat. Acta physiol. scand., 1952, 27, Sppl. 100, 1-67.

16) Okuda, J., Taira, N. \& Motokawa, K. Spectral response curves of postgeniculate neurons in the cat. Tohoku J. exp. Med., 1962, 78, 147-157.

17) Committee on Colorimetry. The Science of Color, Optical Society of America, 1953, 385 p. 1953.

18) Granit, R. Sensory Mechanisms of the Retina, Oxford Univ. Press, London, 1947.

19) Granit, R. Receptors and Sensory Perception, Yale Univ. Press, New Haven and London, 1955.

20) Brindley, B.S. The site of electrical excitation of the human eye. J. Physiol. (Lond.), 1955, 127, 189-200.

21) Mita, T., Fujimaki, E. \& Yaegashi, M. Electrostimulation of the human eye by sinusoidal alternating currents of very low frequency. Tohoku J. exp. Med., 1956, $65,45-56$.

22) Motokawa, K., Yamashita, E. \& Ogawa, T. Responses of retinal network to electrical stimulation. Tohoku J. exp. Med., 1959, 71, 41-53.

23) Crapper, D.R. \& Noell, W.K. Retinal excitation and inhibition from direct electrical stimulation. J. Neurophysiol., 1963, 26, 924-947.

24) Ogden, T.E. \& Brown, K.T. Intraretinal responses of the cynamolgus monkey to electrical stimulation of the optic nerve and retina. J. Neurophysiol., 1964, 27, 682-705. 